\title{
Ascending Aortic Aneurysm Presenting as Hoarseness of Voice: Case Report
}

\author{
Md. Nazmul Hasan ${ }^{1}$, Md. Khorshed Ahmed ${ }^{2}$, Md. Mukhlesur Rahman ${ }^{3}$, Abu Sadique Abdullah ${ }^{4}$, Md. Abu Siddique 5 , Sajal \\ Krishna Banerjee 6
}

\section{ABSTRACT}

Symptomatic thoracic aortic aneurysms (TAA) are rare. A 70 year-old man was admitted with hoarseness of voice for the last six months. Postero-anterior chest X-ray showed left hilar enlargement. Computerised thorax tomography (CTT) images were taken and a saccular TAA $8 \mathrm{~cm}$ in diameter was found. In this paper, we aimed to show that TAA should be considered as a differential diagnosis of patients with hoarseness of voice and hilar enlargement.

Key Words: Thoracic aortic aneurysm, hoarseness of voice.

\section{INTRODUCTION}

Aneurysm is defined as the irreversible dilation of the artery by more than $50 \%$ of the normal diameter. ${ }^{1}$ The rate of thoracic aortic aneurysm (TAA) is 4.5 per hundred thousand people. Frequently, atherosclerosis is a condition manifested by hypertension-induced arterial wall weakening and rupture. Genetic diseases (Marfan syndrome, Loeys-Dietzs syndrome, Ehler Danlos syndrome, familial thoracic aortic aneurysm syndrome, and aneurysm-osteoarthritis syndrome), cystic medial necrosis, giant cell arteritis, infections (syphilis, mycotic infections, tuberculosis) and trauma play a role in the aetiology. ${ }^{2}$ Thoracic aortic aneurysm is usually asymptomatic and is normally diagnosed with imaging techniques performed for unrelated reasons. ${ }^{3}$ Patients with TAA have often had some life-threatening complications, such as aortic rupture, dissection and mortality. In this paper we aimed to present a rare case of TAA found in a 70 year-old male patient presenting with hoarseness of voice.

\section{CASE REPORT}

A 70 year-old man was admitted to our department suffering from hoarseness of voice for six months. It was gradual onset and slowly progressive, associated with dry cough. He also complains of chest pain on exertion for same duration. Pain was compressive in nature, radiates to back and relieved on rest. He had no history of smoking, hemoptysis, dyspnoea, dysphagia, cold intolerance, any weight change and fever. He had also been suffering from hypertension for 20 years with an irregular antihypertensive treatment.

\section{Authors'Information:}

1. Dr. Md. Nazmul Hasan, Resident, Department of Cardiology, Bangabandhu Sheikh Mujib Medical University Dhaka, Bangladesh

2. Dr. Md. Khorshed Ahmed, Associate Professor, Department of Cardiology, Bangabandhu Sheikh Mujib Medical University, Dhaka, Bangladesh

3. Dr. Md. Mukhlesur Rahman, Associate Professor, Department of Cardiology, Bangabandhu Sheikh Mujib Medical University, Dhaka, Bangladesh

4. Dr Abu Sadique Abdullah, Medical Officer, Department of Cardiology, Bangabandhu Sheikh Mujib Medical University, Dhaka, Bangladesh

5. Dr. Md Abu Siddique, Professor \& Chairman, Department of Cardiology, Bangabandhu Sheikh Mujib Medical University, Dhaka, Bangladesh

6. Dr. Sajal Krishna Banerjee, Professor, Department of Cardiology, Bangabandhu Sheikh Mujib Medical University, Dhaka, Bangladesh

Address of Correspondance: Dr. Md. Nazmul Hasan, Resident, Department of Cardiology, Bangabandhu Sheikh Mujib Medical University, Shahbag, Dhaka, Bangladesh. Cell: +880 1675755638,E-mail: nazmulhasan45cmc@gmail.com 
Aetiopathogenetic investigation of the patient revealed no systemic connective tissue disease, infection, genetic defects, inflammation, or history of trauma. Moreover, he did not have any known genetic disease; however, his parents had suffered from hypertension and diabetes. His vital signs were as follows: blood pressure: $140 / 80 \mathrm{~mm} \mathrm{Hg}$, pulse rate: 90 beats/minute, respiratory rate: 16 breaths/minute, body temperature: $36^{\circ} \mathrm{C}$.

Cardiac and other system examinations were normal, but there was a decrease of brea the sounds in the left infrascapular area in the auscultation. Complete blood count, biochemical and serological analyses were normal. The patient had a normal erythrocyte sedimentation rate of $10 \mathrm{~mm} / \mathrm{h}$ and a white blood cell count of $9 \times 10^{9} / \mathrm{L}$. High sensitivity C-reactive protein and serum Ddimer levels were found to be normal. Poster anterior chest $\mathrm{X}$ - ray examination revealed a large left hilar mass. In addition to a lack of aeration of the lower lobe of the left lung, there was minimal costophrenic sinus bluntness.

In echocardiographic examination, systolic function was normal (fractional shortening: $30 \%$, ejection fraction:65\%), there was grade I diastolic dysfunction, no valvular regurgitation and hypertrophy (inter ventricular septum diastolic diameter: $10 \mathrm{~mm}$ ). Calcification existed in the aortic lid. Haemodynamically insignificant atheroma plaques were detected in bilateral carotid arteries by color and pulse wave Doppler ultrasonoghraphy. Computerized thoracic tomography scan showed that the mass was located in the proximal part of the ascending aorta, with a diameter of $8 \mathrm{~cm}$, suggesting a saccular aortic aneurysm. Defined thrombus material was pressurizing the posterior of the esophagus and the trachea. Also, due to compression, atelectasis was seen on the posterobasal segment of the left lung. Additionally, a large thrombus $(6 \mathrm{~cm})$ and atherosclerotic atheroma plaques were seen within the TAA (Figure $1 \& 2$ ). There was no pleural effusion. Thoracic aortography examination showed an aneurysm located in the proximal part of the ascending aorta with a diameter of $8 \mathrm{~cm}$. Then the patient was referred to Cardiac surgery Department for surgical repair.

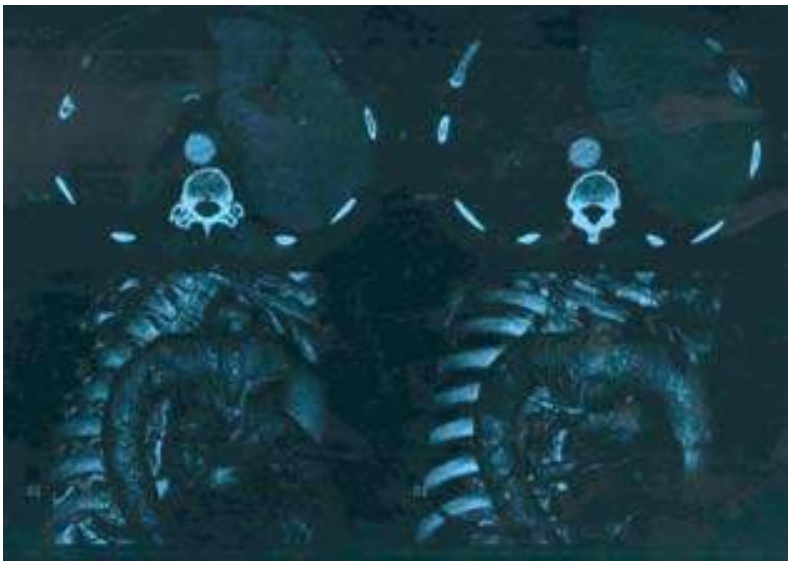

FIGURE 1: CT Scan of Chest with contrast showing Partially thrombosed aneurysm of ascending aorta

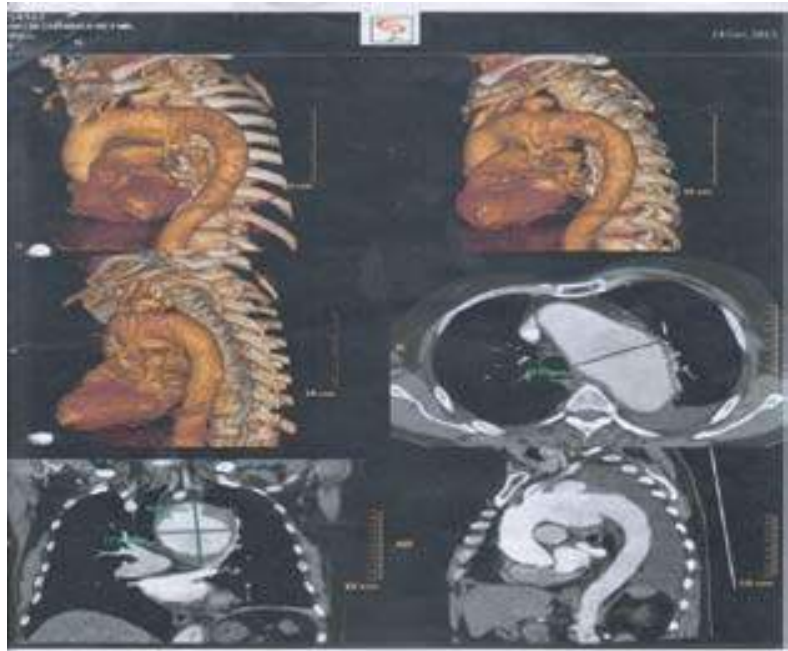

FIGURE 2: Different views of CT Scan of Chest with contrast showing aneurysm involving ascending aorta

\section{DISCUSSION}

Rupture of TAA and dissections are very rare, despite the very high morbidity and mortality rates. Therefore, early detection is important. Thoracic aortic aneurysms are usually asymptomatic (about $75 \%$ ), but pain is known 
as the predominant referable symptom in about $17 \%$ of patients. Although usually asymptomatic, chest pain, back pain, hoarseness due to recurrent laryngeal nerve compression, difficulty in swallowing due to compression of the esophagus and shortness of breath due to the bronchial compression may be seen. ${ }^{4}$ In our case, there was no continuity in back pain over the last two years. Hilar enlargement on chest radiographs suggested the need for a computerized thorax tomography scan, which revealed the descending TAA. The rate of descending aortic aneurysms in the etiology of atherosclerosis is high. 5,6

In addition in the ascending aorta, the aneurysms may be saccular or of fuzzy form and may press on to the esophagus and trachea and may present as dysphasia and hoarseness of voice. Moreover, in the real aneurysms, all layers of the aorta wall may be affected. Usually, pseudo aneurysms may occur after trauma or as a post-operative complication. ${ }^{7}$ In our case, a thoracic saccular aneurysm was seen, which spanned all layers of the aorta. No dysphasia was observed, but hoarseness of voice as there was pressure on the trachea in CTT.

In our case, the patient had no genetic or systemic disease, no infection, and no history of trauma. Atheromatous plaque findings in CTT also suggested an aneurysm. It is unknown whether atherosclerotic aneurysms are caused by atherosclerosis or atherosclerosis is caused by deformities in vascular structure caused by aneurysms; however, we believe in our case that the atherosclerosis is associated with the thoracic aortic aneurysm. In aneurysms, smoking history, chronic obstructive pulmonary disease, advanced age, pain, hypertension, and a diameter of more than $5 \mathrm{~cm}$ of the aorta increases the risk of aortic rupture. ${ }^{7}$ In the case presented here, despite an aortic diameter of $8 \mathrm{~cm}$, advanced age and hypertension, the lack of rupture positively influenced the prognosis of the patient's diagnosis. Now a days, because of low morbidity, mortality and hospital stay, thoracic endovascular stent graft surgery, generally under epidural anesthesia, is the preferred surgical method in especially old TAA patients. $8,9,10$

Thoracic endovascular stent graft surgery was applied to our patient too. The lack of postoperative complication suggests that endovascular stent graft surgery in TAA without rupture or dissection will diminish mortality rates. Consequently, we believe that, despite its rare incidence, TAA should not be forgotten in the differential diagnosis of hoarseness of voice. In these patients, early diagnosis diminishes mortality rates and increases the quality of life.

\section{REFERENCES}

1. Coady MA, Rizzo JA, Goldstein LJ, Elefteriades JA Natural history, pathogenesis, and etiology of thoracic aortic aneurysms and dissections. Cardiol Clin 1999; $17(4): 615-35$.

2. Isselbacher EM. Thoracic and abdominal aortic aneurysms. Circulation 2005;111:816-28.

3. Akin I, Kische S, Rehders TC, Nienaber AC, Rauchhaus $M$, Ince $H$. Endovascular repair of thoracicaortic aneurysm. Arch Med Sci 2010;6(5):646-52.

4. Buket S, Bilgen F, Battalolu B, Gürbüz A, Alhan C. Aortcerrahisindetan vetedaviklavuzu 2008. TürkKalp Damar Cerrahisi Dernei Aort Cerrahisi Çalisma Grubu; 2008.

5. Joyce WJ, Fairbairn JF, Kincaid DW, Juergens JL. Aneurysms of the thoracic aorta. A clinical study with special reference to prognosis. Circulation 1964;29: 176-81.

6. Findeis LK, Cody ME. Endovascular repair of thoracic aneurysms. Semin Intervent Radiol 2011;28:107-17.

7. Duru S, Erdem M, Agca E, Kaplan T, Ardiç S. Thoracic Aortic Aneurysm: A Rare Case Report. Turk Toraks Derg 2013;14:78-80. DOI: 10.5152/ttd.2013.16

8. Hountis PG, Plestis KA. Strategies in the management of extensive descending and thoracoabdominal aortic aneurysms. Hellenic J Cardiol 2009;50:295-302.

9. Bortone AS, De Cillis E, D'Agostino D, Schinosa LLT. Endovascular treatment of thoracic aortic disease: four years of experience. Circulation 2004;110:262-7.

10. Stone DH, Brewster DC, Kwolek CJ, Lamuraglia GM, Conrad MF, Chung TK et al. Stent-graft versus opensurgical repair of the thoracic aorta: mid-term results. J Vasc Surg 2006;44:1188-97. 